\title{
Disturbance Error Correction Algorithm for UAV Flight Control Based on Adaptive Backstepping Integral
}

\author{
Xing-Hua Lu ${ }^{1, a}$, Shi-wei Zhan ${ }^{1, b}$,Wen-quan Yu ${ }^{1, c}$, Chen-wei Huang ${ }^{1, \mathrm{~d}}$ \\ ${ }^{1}$ Huali College Guangdong University of Technology, Guangzhou Zengcheng, 511325, P. R. China \\ a44680189@qq.coma, ${ }^{b} 758756766 @ q q . c o m,{ }^{c} 1009182611 @ q q . c o m,{ }^{d} 495962693 @ q q . c o m$
}

Keywords: UAV, control,error correction, adaptive.

\begin{abstract}
The gas dynamic features of Unmanned Aerial Vehicle (UAV) cause flight attitude control error in aerodynamic disturbances, it need for stability flight control design of UAV, to improve the UAV flight stability property. Under the condition of large disturbance, the uncertainty of parameters of flight control model is caused by the drastic change of the air resistance and other parameters of the UAV. An error correction algorithm for UAV flight control is proposed based on adaptive backstepping integral. UAV longitudinal motion model is established, and the UAV control parameter constraint model is established in velocity coordinate system and body coordinate system, ballistic coordinate and ground coordinate system. The longitudinal motion mathematical model of UAV is established. According to the principle of Lyapunove stability control, adaptive nonlinear tracking controller is designed. On the output terminal, adaptive inversion integral term is introduced, and the global asymptotic tracking error compensation is taken to improve the stability of flight control. Simulation results show that the proposed algorithm can effectively improve adaptive guidance performance of flight control, and the attitude angle error converges to zero, and the performance is better.
\end{abstract}

\section{Introduction}

UAV is the aircraft with the radio control equipment and control devices owned by the program control of unmanned aircraft. With the development of aircraft design and manufacturing technology, the production and design techniques of UAV get comprehensive promotion, and UAV has been widely used in the field of aerospace, target recognition, intelligent detection and field investigation. UAV has the characteristics such as convenience and low cost investment, it shows a good application prospect in the future military and civilian fields ${ }^{[1]}$.

UAV flight stability control is the key technology which restrict the development of the machine, in the vertical flight plane of UAV, due to flight attitude angles change on a large scale and effect of the airflow, resulting in body trembling, affect the flight stability. It is significant to improve the stability and reliability of UAV flight control algorithm, and it has obtained great importance to the research of the algorithm. UAV flight stability control is an important bottleneck technology in the development of UAV, the gas dynamic characteristic has static instability, in the longitudinal plane, it is affected by the continuous change of the air density and air flow, and other flight conditions, leads to the UAV cannot make a stable flight, flight trajectory deviation is appeared, which caused by UAV roll and chattering, it even can appear the flight accidents ${ }^{[2-4]}$.

Traditionally, the UAV flight control algorithms are mainly researched based on Fuzzy Neural Network PID control of flight control algorithm, the particle swarm optimization algorithm for UAV flight control algorithm and adaptive noise cancellation of flight control algorithm, bionic intelligent group control of flight control algorithm. The algorithms in the whole flight envelope will cause nonlinear mathematical model under a particular flight condition with least squares fitting processing, and improve the stability of the UAV flight attitude control, but traditional methods with interpolation or fitting method, and the parameters of controller in a finite number of linear model between continuous variation, it leads to attitude angle error tracking process, and it is prone to distortion, cause a disturbance of the error ${ }^{[5,6]}$. 
In view of the above problems, it needs for stability flight control design of UAV, to improve the UAV flight stability property.An error correction algorithm for UAV flight control is proposed based on adaptive backstepping integral. Firstly, the control motion model of UAV is constructed, the construction flight reference coordinate system is taken, and the object of mathematical model analysis is carried out, on the basis of this, build UAV flight control constraint parameter model, according to the principle of Lyapunove stability control, the adaptive nonlinear tracking controller is designed. On the output terminal, adaptive inversion integral term is introduced, and the global asymptotic tracking error compensation is taken to improve the stability of flight control. Simulation results show that the proposed algorithm can effectively improve adaptive guidance performance of flight control, the conclusion is drawn, and it shows the good application value.

\section{Motion model construction and control object description of UAV flight control}

\subsection{Construction of motion model for flight control of UAV}

In order to realize the UAV flight control optimization and disturbance error correction, first of all, we should build a UAV flight control of the motion model, UAV attitude control has the properties of time-varying of parameters and disturbances of random variation, to construct a deterministic model to describe the controlled object, he parameters of the object is constructed and a description, flight attitude control parameters include two parts as the deterministic parameters and uncertain parameters, uncertain parameters mainly include parameter uncertainty and external disturbance, based on the above analysis, the establishment of UAV longitudinal motion model is described as follows:

In this paper, we mainly use the following coordinate system: velocity coordinate system, body coordinate system, ballistic coordinate system, and ground coordinate system.

1) Elocity coordinate system $O_{3} y_{3} z_{3}$, the centroid of UAV is the origin of the coordinate system $O$; selecting velocity vector $V$ as $O x_{3}$ axis; $O y_{3}$ axis is vertical to $O x_{3}$ axis, and it is located in the UAV longitudinal symmetry plane, the above is positive; $\mathrm{Oz}_{3}$ axis is vertical to $O x_{3} y_{3}$ plane, the direction is determined by the right-hand rule.

2) Body coordinate system $O x_{1} y_{1} z_{1}$. The body coordinate system is connected to UAV.

The centroid of UAV is the origin of coordinates $O, O x_{1}$ points to the head. It is coincident with the UAV longitudinal axis, $O y_{1}$ is positive, in the plane of the longitudinal symmetry plane, $O z_{1}$ is determined by right-hand rule.

3) Ballistic coordinate system $\mathrm{Ox}_{2} y_{2} z_{2}$. The centroid of UAV is the origin of the coordinate system, selecting velocity vector $V$ as $\mathrm{Ox}_{2}$ axis, $\mathrm{Oy}_{2}$ axis is located in a vertical plane, the plane contains the velocity vector, $\mathrm{Oz}_{2}$ is determined by right-hand rule.

4) The ground coordinate system Axyz. The ground coordinate system Axyz is a coordinate system which is fixedly connected with the earth. UAV centroid in the ground projection as the coordinates origin, Ay axis is vertical to axis。Ay axis is determined by right-hand rule.

According to the flight control coordinate system construction of UAV flight control, the motion model is constructed. Assumed that the UAV longitudinal motion is symmetrical and in longitudinal motion, UAV tilt control mechanism and a yaw control mechanism without action, in order to reduce the risk coefficient of UAV flight, when the roll motion process of UAV happened, flight error correction is taken, and it can be obtained individually UAV longitudinal motion equations described as:

$$
\mathbf{z}(\mathbf{k})=\mathbf{b}(\mathbf{k}) s(k)+\mathbf{n}(\mathbf{k}), k \in Z
$$

In formula, $\mathbf{b}(\mathbf{k}), s(k), \mathbf{n}(\mathbf{k})$ respectively are the channel vector of the total aerodynamic force acting on the UAV, the inertial product of the UAV of the body coordinate system $\mathrm{Oz}_{1}$, and the noise vector. 
The weighted combination contribution of each point source of the centroid acceleration in the vertical plane $\mathrm{Ox}_{2} y_{2}$ of the UAV can be described as:

$$
\mathbf{b}(\mathbf{k})=\int_{-\pi}^{\pi} f(\theta, k) \mathbf{a}(\boldsymbol{\theta}) d \theta
$$

Where, $\mathbf{a}(\boldsymbol{\theta})=\left[1, e^{-j \pi \sin \theta}, e^{-j 2 \pi \sin \theta}, \ldots e^{-j(M-1) \pi \sin \theta}\right]^{T}$ is a conventional $M \times 1$ order point source array flow pattern vector, $f(\theta, k)$ is a time weighted function of the longitudinal motion of the UAV. It is a time weighted function of the longitudinal motion of the UAV, which describes the variation of the scattering components from each angle with time. About UAV flight angle time weighting function: for each direction $\theta$, the angle time weighting function can be understood as a random process, according to this, there are the following assumptions:

1) The tilt control mechanism of the UAV and the yaw control mechanism have no action, at this time, the mean value of the disturbance error is:

$$
E[f(\theta, k)]=0, \forall \theta \in[-\pi, \pi], \forall k \in Z
$$

2) Statistical independence of each angle in the trajectory, the certain feature points are:

$$
P_{f f^{\prime}}\left[f(\theta, k), f\left(\theta^{\prime}, k^{\prime}\right)\right]=P_{f}[f(\theta, k)] P_{f^{\prime}}\left[f\left(\theta^{\prime}, k^{\prime}\right)\right], \theta \neq \theta^{\prime} \forall k, k^{\prime} \in Z
$$

3) The UAV flight control process is a generalized time stationary process, it is shown as:

$$
E\left[f(\theta, k), f^{*}\left(\theta^{\prime}, k^{\prime}\right)\right]=E\left[f(\theta, k+l), f^{*}\left(\theta^{\prime}, k^{\prime}+l\right)\right], \forall k, k^{\prime}, l \in Z
$$

It can be seen that the longitudinal motion equation of the UAV is a set of dynamic systems composed of nonlinear differential equations. To study the perturbation motion requires the solution of nonlinear differential equations, due to the complexity of the flight environment, in most cases it is multipath channel, when the geometric distribution is completely random, such as flight arrival phase control of every direction and the receiving array are completely independent, the robustness of the UAV flight is limited. In addition, in the relatively short observation interval and it needs to use a weighted correlation function, the function that depends only on the discrete time interval $l$, usually a small disturbance stability is taken as the premise, derive the UAV linear perturbation equations as:

$$
E\left[f(\theta, k) f^{*}\left(\theta^{\prime}, k+l\right)\right]=\gamma_{f}(\theta, l) \delta\left(\theta-\theta^{\prime}\right), \forall l \in Z
$$

Where, $\gamma_{f}(\theta, l)$ is the correlation function of $f(\theta, k)$, it is usually called the angle time correlation kernel (ATCK). On the basis of the analysis and description of the constraint parameters and the controlled object of the UAV flight, control algorithm design is carried out.

\subsection{Control parameters of the UAV flight control and the description of the controlled object}

UAV flight control object is a closed control system. In non zero delay longitudinal motion, disturbance error is zero, assuming that the observed signal is $\mathrm{N} \times 1$ dimensional random vector, additive noise is Gaussian white noise, the signal is between noise and irrelevant. The parameters of robust control and adaptive control are $\boldsymbol{\eta}=\left[\psi_{0}, \sigma_{\psi}, \sigma_{s}{ }^{2}, \sigma_{n}{ }^{2}\right]^{T}$, These parameters can be obtained by minimizing the following functions: $\hat{\boldsymbol{\eta}}=\arg \min _{\eta} L(\boldsymbol{\eta})$, and:

$$
L(\boldsymbol{\eta})=\ln \operatorname{det} \mathbf{R}_{\mathbf{x}}(\boldsymbol{\eta})+\operatorname{trace}\left(\mathbf{R}_{\mathbf{x}}^{-\mathbf{1}}(\boldsymbol{\eta}) \hat{\mathbf{R}}_{\mathbf{x}}\right)
$$

In formula, $\mathbf{R}_{\mathbf{x}}(\boldsymbol{\eta})=\mathbf{R}_{\mathbf{s}}\left(\boldsymbol{\psi}_{\mathbf{0}}, \boldsymbol{\sigma}_{\boldsymbol{\psi}}, \boldsymbol{\sigma}_{\mathrm{s}}{ }^{2}\right)+\sigma_{n}{ }^{2} \mathbf{I}, \mathbf{R}_{\mathbf{x}}$ is the centroid acceleration covariance matrix, and $\mathbf{R}_{\mathrm{s}}$ is the signal covariance matrix, $\sigma_{n}{ }^{2}$ is the energy of the disturbance error of the centroid acceleration along the trajectory, $\hat{\mathbf{R}}_{\mathbf{x}}$ is the variance matrix of the sampling data, $\hat{\mathbf{R}}_{\mathbf{x}}=\frac{1}{K} \sum_{k=1}^{K} \mathbf{x}_{\mathbf{k}} \mathbf{x}_{\mathbf{k}}{ }^{\mathbf{H}} \cdot K$ is the number of snapshots. Adaptive controller structure is similar to the robust controller. During the flight, assumed successive snapshots are statistically independent. 
According to the above analysis, the covariance matrix of the closed loop system of the controller of the UAV flight control system can be expressed as:

$$
\mathbf{R}=E\left[\mathbf{z}(\mathbf{t}) \mathbf{z}^{\mathbf{H}}(\mathbf{t})\right]=\mathbf{B P}_{\mathbf{s}} \mathbf{B}^{\mathbf{H}}+\sigma_{n}^{2} \mathbf{I}_{\mathbf{M}}
$$

Where, $\mathbf{B}=\left[b_{1}\left(\theta_{1}\right), b_{2}\left(\theta_{2}\right), \ldots, b_{q}\left(\theta_{q}\right)\right]^{T}$, the singular value vector matrix of the controlled object is decomposed into:

$$
\mathbf{R}=\mathbf{U}_{\mathrm{s}} \boldsymbol{\Lambda}_{\mathrm{s}} \mathbf{U}_{\mathrm{s}}^{\mathrm{H}}+\mathbf{U}_{\mathbf{n}} \boldsymbol{\Lambda}_{\mathbf{n}} \mathbf{U}_{\mathbf{n}}^{\mathrm{H}}
$$

The column vectors of the matrixes $\mathbf{U}_{\mathbf{s}}$ and $\mathbf{U}_{\mathbf{n}}$ are composed of singular values $\sigma_{1}, \sigma_{2}, \ldots \sigma_{q}$, and $\sigma_{n}$, corresponding singular vectors. In the control system, there is a stable parameter scheduling, then $\sigma_{1} \geq \sigma_{2} \geq \ldots \geq \sigma_{q}>\sigma_{n}$, which assumes that there is no coherence between the different distribution sources, then $\mathbf{U}_{\mathbf{n}}^{\mathbf{H}} b_{i}\left(\theta_{i}\right)=0$. The control error of the control information transmission control is completely compensated by the feature extraction. With this point, we can obtain multidimensional spectrum peak search method of the robust control object in the UAV flight control system:

$$
f(\phi)=\frac{1}{\mathbf{b}^{\mathbf{H}}(\varphi) \hat{\mathbf{U}}_{\mathbf{n}} \hat{\mathbf{U}}_{\mathbf{n}}^{\mathbf{H}} \mathbf{b}(\varphi)}
$$

Based on the analysis of the steady motion of the UAV flight, the controller design is optimized, and the controller is designed.

\section{Control disturbance error correction algorithm and controller optimization design based on adaptive backstepping integral}

On the basis of the controlled object description and control parameters analysis, the controller design is optimized. In this paper, an error correction algorithm for flight control is proposed based on adaptive backstepping. The longitudinal motion model of UAV is established, and the mathematical model of longitudinal motion of UAV is obtained. Lyapunove stability control principle is designed, and the adaptive nonlinear tracking controller is designed, UAV flight control problem is formulated for the solution for minimization problems as follows:

$$
\left(\theta_{i}, \sigma_{i}\right)=\arg \min \mathbf{b}_{\mathbf{i}}^{\mathbf{H}}(\boldsymbol{\theta}, \boldsymbol{\sigma}) \hat{\mathbf{U}}_{\mathbf{n}} \hat{\mathbf{U}}_{\mathbf{n}}^{\mathbf{H}} \mathbf{b}_{\mathbf{i}}(\boldsymbol{\theta}, \boldsymbol{\sigma})
$$

The tracking error of the angular velocity of the UAV flight control is obtained, and the sliding mode surface is satisfied in the geometric control region of the UAV flight control:

$$
\mathbf{b}_{\mathbf{i}}\left(\boldsymbol{\theta}_{\mathbf{i}}\right)=\boldsymbol{\Phi}\left(\boldsymbol{\theta}_{\mathbf{i}}\right) \mathbf{h}_{\mathbf{i}}
$$

Where, M order diagonal matrix $\boldsymbol{\Phi}\left(\boldsymbol{\theta}_{\mathbf{i}}\right)=\operatorname{diag}\left(\mathbf{a}\left(\boldsymbol{\theta}_{\mathbf{i}}\right)\right)$, M order vector $\mathbf{h}_{\mathbf{i}}=\int_{-\pi}^{\pi} \mathbf{a}(\boldsymbol{\theta}) g_{i}(\theta) d \theta$, it can be proved that if the distribution function of the velocity in the longitudinal motion is a conjugate symmetric function, then, $g_{i}(\theta)=g_{i}^{*}(-\theta)$, vector $\mathbf{h}_{\mathbf{i}}$ is real vector, $\mathbf{h}_{\mathbf{i}} \in R^{M}$. Under the distributed target parameter estimation, the dynamic coefficients, and the transfer function and the frequency domain characteristics of the UAV are solved:

$$
\left(\theta_{i}, \sigma_{i}\right)=\arg \min \mathbf{h}_{\mathbf{i}}^{\mathrm{H}} \boldsymbol{\Phi}^{\mathbf{H}}(\boldsymbol{\theta}) \hat{\mathbf{U}}_{\mathbf{n}} \hat{\mathbf{U}}_{\mathbf{n}}^{\mathrm{H}} \boldsymbol{\Phi}(\boldsymbol{\theta}) \mathbf{h}_{\mathbf{i}}
$$

Assumed $\mathbf{Q}_{\mathbf{1}}(\boldsymbol{\theta})=\boldsymbol{\Phi}^{\mathbf{H}}(\boldsymbol{\theta}) \hat{\mathbf{U}}_{\mathbf{n}} \hat{\mathbf{U}}_{\mathbf{n}}^{\mathbf{H}} \boldsymbol{\Phi}(\boldsymbol{\theta})$, for the steady state error of the flight attitude boundary layer, the minimum eigenvalue of the matrix $\mathbf{Q}_{\mathbf{1}}(\boldsymbol{\theta})$ can be used to compensate the error. By introducing the adaptive inversion integral term, the spectral peak position of the spatial spectrum is obtained, and the output error is obtained:

$$
f_{1}(\theta)=-\log 10\left(\lambda_{\min }\left[\mathbf{Q}_{1}(\boldsymbol{\theta})\right]\right)
$$


Above all, we can use this method to modify the disturbance error of flight control, the adaptive inversion integral is introduced to compensate the global asymptotic tracking error, and improve the stability of flight control.

\section{Simulation experiment and result analysis}

In order to verify the control performance of the algorithm, the simulation experiment is carried out. First of all, we build hardware platform of a flight control simulation, flight attitude data are collected. Using Matlab programming, control algorithm design is obtained. The arrival directions of the UAV fuselage flutter source are $\theta_{1}=-15^{0}, \theta_{2}=10^{\circ}$, The pitch angles of UAV flight are $\Delta_{1}=5^{0}, \Delta_{2}=8^{0}$, Signal to noise ratio is 0 . The number of snapshots is 1000 , adaptive parameter $\varepsilon_{1}=0.1$. Based on the simulation environment and parameters setting, the simulation of UAV flight control and disturbance error compensation are carried out, the UAV flight angle scanning results are shown in Figure 1.

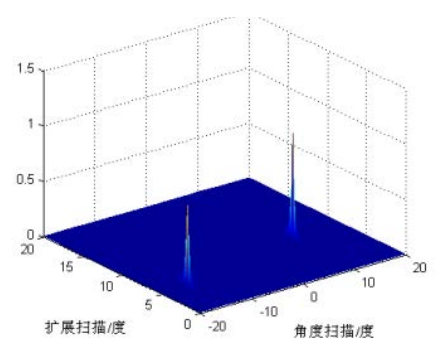

Figure 1 UAV flight angle scanning results

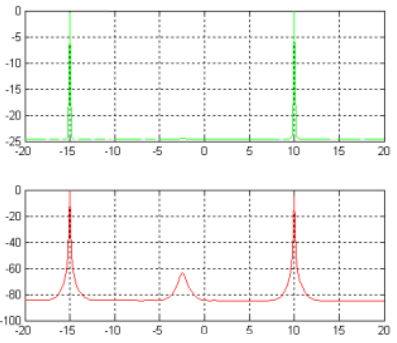

Figure 2 Output spatial spectrum of UAV flight control

From figure 1 it can be seen, the UAV flight control is on the conditions of large disturbance, due to the intense change without man-machine air resistance parameters, resulting in disturbance error, resulted in attitude angle control distortion, using the method for correction, and the output spatial spectrum with correction control is shown in Figure 2.

using the method of perturbation error correction, correction control output spatial spectrum as shown

The control convergence curve is shown in Figure 3, from figure it can be seen, the new method can effectively improve adaptive guidance performance, the attitude angle error converges to zero, it has better performance.

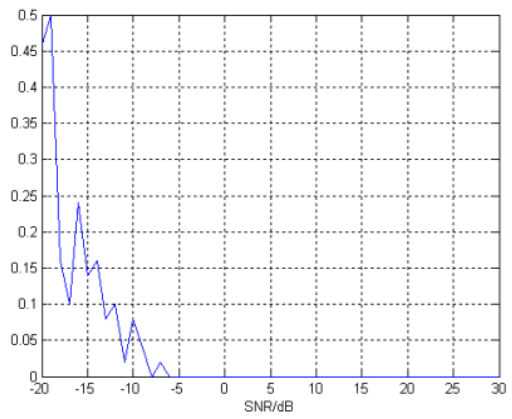

Figure 3. Control convergence curve

\section{Conclusions}

In this paper, an error correction algorithm for UAV flight control is proposed based on adaptive backstepping integral. UAV longitudinal motion model is established. According to the principle of Lyapunove stability control, adaptive nonlinear tracking controller is designed. The global 
asymptotic tracking error compensation is taken to improve the stability of flight control. Simulation results show that the proposed algorithm can effectively improve adaptive guidance performance of flight control, and the attitude angle error converges to zero, and the performance is better.

\section{Acknowledgments}

This project is supported by Foundation for Distinguished Young Talents (Natural Science, 2015KQNCX218) of 2015 Key Platforms and Research Projects of Department of Education of Guangdong Province and 2016 Undergraduate Scientific and Technological Innovation Project Fund of Guangdong Province (pdjh2016b0940); "Mechanical and Electrical Integrated Skills Training Center" of Quality Projects in Guangdong Province(Official Letter No.204 from Higher Education of Department of Education of Guangdong in 2012)

\section{References:}

[1] ZHAO Shilei, GUO Hong, LIU Yupeng. Fault Tolerant Control for Linear Time-delay System Based on Trajectory Tracking[J]. Information and control, 2015,44(4): 469-473.

[2] MING Ping-song LIU Jian-chang. Consensus stability analysis of stochastic multi-agent systems. Control and Decision, 2016, 31(03): 385-393.

[3] Li T, Zhang J. Consensus conditions of multi-agent systems with time-varying topologies and stochastic communication noises[J]. IEEE Trans on Automatic Control, 2010, 55(9): 2043-2057.

[4] Mahmoud E E. Complex complete synchronization of two nonidentical hyperchaotic complex nonlinear systems[J]. Mathematical Methods in the Applied Sciences, 2014, 37(3): 321-328.

[5] Palomares I, Martinez L, Herrera F. A consensus model to detect and manage non-cooperative behaviors in large scale group decision making[J]. IEEE Trans on Fuzzy System, 2014, 22(3): 516-530.

[6] DONG Bo LIU Ke-ping LI Yuan-chun. Decentralized integral sliding mode control for time varying constrained odular and reconfigurable robot based on harmonic drive transmission. Control and Decision, 2016, 31(03): 441-447. 